\title{
Das Partherreich als Bindeglied der Seidenstraße zwischen China und Rom
}

\author{
Bettina Darlap \\ Kerngebiet: Alte Geschichte \\ eingereicht bei: assoz. Prof. Mag. Dr. Brigitte Truschnegg \\ eingereicht im: WiSe 2017/18 \\ Rubrik: BA-Arbeit
}

\begin{abstract}
The Parthian Empire as an Intermediary between China and Rome in the Silk Road Trade

This paper analyses the role of the Parthian Empire in the Silk Road trade between China and the Roman Empire. It examines Parthian, Roman and Chinese sources to achieve a better understanding of its importance for trade contacts along the Silk Road. As the analysis will show, Roman and Chinese sources differ greatly in their interpretation of the role the Parthians played within these trade networks, and they shed light on different aspects of the relationships, be it in trade or otherwise.
\end{abstract}

\section{Einleitung}

In dieser Arbeit soll die Rolle der Parther im Rahmen des Handels der Seidenstraße erörtert werden. Zusätzlich soll untersucht werden, ob sich die Wahrnehmung der Parther als "Intermediär der Seidenstraße" in römischen und chinesischen Quellen unterscheidet. Zu diesem Zweck wird ein Vergleich dieser Quellen vorgenommen.

Die Parther standen neben dem Römischen Reich und Han-China mit diversen anderen Reichen, beispielsweise Baktrien oder Kouchan, in Kontakt. Für ein abschließendes Verständnis der Rolle der Parther im Handel der Seidenstraße wäre es daher notwendig, auch ihr Verhältnis zu diesen Reichen in die Analyse aufzunehmen. Dies würde allerdings den Rahmen der Arbeit sprengen. Die Arbeit konzentriert sich daher 
auf die beiden wichtigsten Imperien ihrer Zeit - Rom und China. Die vorhandenen Schriftquellen werden kritisch analysiert und durch Erkenntnisse aus archäologischen oder numismatischen Untersuchungen ergänzt. Wichtige Arbeiten zu den Parthern, den vorhandenen Quellen, dem Fernhandel und der Seidenstraße wurden unter anderem von Ursula Hackl u. a. (2010)', Josef Wiesehöfer und Sabine Müller (2017)², Gary K. Young $(2011)^{3}$ und Emmanuel Choisnel (2004) $)^{4}$ verfasst.

Zu Beginn dieser Arbeit wird der Begriff der Seidenstraße näher erörtert und ein kurzer Überblick über die Geschichte des Partherreichs gegeben, um das Thema genauer zu verorten. Im folgenden Kapitel werden die vorhandenen Primärquellen und ihre Verfasser vorgestellt und ihre Glaubwürdigkeit kritisch hinterfragt, um möglicherweise inhärente Probleme und Wertungen zu akzentuieren. Im Hauptteil der Arbeit werden ausgewählte Quellen im Detail analysiert und in Bezug auf die Fragestellung gesetzt. Hierbei werden Ergebnisse der Sekundärliteratur ergänzend herangezogen. Es folgt ein Vergleich der aus den römischen und chinesischen Quellen gewonnenen Informationen. Die Arbeit schließt mit einem Ausblick auf weitere mögliche Untersuchungsthemen.

\section{Die Seidenstraße}

Bei der Bezeichnung Seidenstraße handelt es sich letztlich um einen anachronistischen Begriff, der in parthischer Zeit noch nicht verwendet wurde, sondern erst Ende des 19. Jahrhunderts durch den Geographen Ferdinand Freiherr von Richthofen geprägt wurde. Die Seidenstraße bestand auch nicht aus einer einzelnen Route; vielmehr wurde eine Vielzahl von Wegen zu Land und zu Wasser genutzt. ${ }^{5}$ Bei diesen Reisewegen handelte es sich außerdem nicht primär um befestigte Straßen, sondern oft um nicht gekennzeichnete Wege, die sich den jeweiligen politischen wie geographischen Gegebenheiten anpassten. ${ }^{6}$ Zusätzlich muss davon ausgegangen werden, dass es sich bei der Seidenstraße um ein Handelsnetzwerk handelte, das nicht vorrangig die „Endpunkte" HanChina und das Römische Reich verband, sondern vielmehr den Handel zwischen allen Ländern einschloss, die an dieser Route lagen. Gerade die Nachfrage dieser oft als Zwischenstationen betrachteten Länder prägte die Auswahl der gehandelten Waren stark.

1 Ursula Hackl, Das Partherreich und Rom seit dem 1. Jh. v. Chr., in: Ursula Hackl/Bruno Jacobs/Dieter Weber (Hrsg.), Quellen zur Geschichte des Partherreiches. Textsammlung mit Übersetzungen und Kommentaren, Bd. 1 (Novum Testamentum et orbis antiquus 83), Göttingen u. a. 2010, S. 56-77; Ursula Hackl, Handel und Wirtschaft, in: Hackl/ Jacobs/Weber (Hrsg.), Quellen, S. 111-124; Ursula Hackl, Schriftquellen zur Geschichte des Partherreichs, in: Hackl/ Jacobs/Weber (Hrsg.), Quellen, S. 21-30.

2 Sabine Müller, Das Bild der Parther bei Trogus-Justin, in: Sabine Müller/Josef Wiesehöfer (Hrsg.), Parthika. Greek and Roman Author's Views of the Arasacid Empire (Classica et Orientalia 15), Wiesbaden 2017, S. 241-257.

3 Gary K. Young, Rome's Eastern Trade. International Commerce and Imperial Policy 31 BC-AD 305, New York 2011.

4 Emmanuel Choisnel, Les Parthes et la Route de la Soie (Collection Centre Asie), Paris 2004.

5 James A. Millward, The Silk Road. A Very Short Introduction, Oxford 2013, S. 3-7.

6 Valerie Hansen, The Silk Road. A New History, Oxford 2012, S. 5. 
Und schließlich war die später namensgebende Seide auch nicht das am meisten gehandelte Gut auf dieser Strecke?

Der Handel wurde selten über die ganze Strecke von einem einzelnen Händler durchgeführt. Vielmehr war er häufig lokaler Natur und wurde oft nicht mit Münzen vergütet, sondern erfolgte in Naturalien. Dass der Handel auf der Seidenstraße nicht primär von römischen Händlern dominiert wurde, wird auch durch die Abwesenheit von römischen Münzen in China bestätigt. Die frühesten in China gefundenen römischen Münzen stammen aus byzantinischer Zeit. ${ }^{8}$ Die Beherrschung des Handels durch einen einzelnen Akteur konnte jedoch ebenfalls der Fall sein, so etwa im Handel des Römischen Reichs mit Indien, dessen direkte Natur sich in Funden von römischen Münzen in Süd- und Zentralindien niederschlug. ${ }^{9}$ Die große Bedeutung, die den Parthern in diesem Handelsnetzwerk zugeschrieben wurde, zeigt sich unter anderem dadurch, dass in der Forschungsliteratur der Beginn des Handels entlang der Seidenstraße oft mit einer chinesischen Gesandtschaft angesetzt wird, die um 115 v. Chr. zu Mithridates II. ${ }^{10}$ kam. Tatsächlich wurde aber schon vor den Parthern, unter den Achaimeniden und Seleukiden, Handel auf diesen Routen betrieben. ${ }^{11}$ Dies wird durch Untersuchungen untermauert, die der Verbreitung von Getreidesorten, Herdentieren und Glas entlang der Seidenstraße nachgingen. Auch metallurgische Analysen legen diese Schlussfolgerung nahe. ${ }^{12}$ Die Sicherung der Handelsstraßen von Afghanistan bis an den Euphrat unter der Herrschaft der Parther ermöglichte aber im dritten Jahrhundert v. Chr. eine Intensivierung der Handelsbeziehungen. ${ }^{13}$ Die Zunahme der Kontakte entlang dieser Handelsstrecken schlug sich auch im kulturellen Bereich nieder. So kann beispielsweise die Entstehung der Terrakottafiguren unter dem ersten Kaiser in China, nach Lukas Nickel, nur im Zusammenhang mit hellenistischen Kulturkontakten verstanden werden. ${ }^{14}$ Kam es zu regionalen oder auch globalen politischen Verwerfungen, wurden diese Handelsrouten auch wieder unterbrochen; die längste Unterbrechung der Landroute durch das Partherreich ist von 8 bis 73 n. Chr. nachweisbar.

\section{Das Partherreich}

Das Partherreich bestand von 250 v. Chr. bis 224 n. Chr. und wurde von der Dynastie der Arsakiden beherrscht. Es handelte sich um ein multikulturelles Großreich, das dezentral organisiert war. ${ }^{15}$ Diese Organisation wurde gerade in römischen Quellen oft als Schwäche ausgelegt. Neuere Forschungsarbeiten zeigen allerdings, dass Wirtschaft und Kultur unter den Arsakiden einen Aufschwung erfuhren, da für diese Zeit keine

Millward, The Silk Road, S. 3-7.

Hansen, The Silk Road, S. 4-9.

Walter Schmitthenner, Rome and India. Aspects of Universal History during the Principate, in: The Journal of Roman Studies 69 (1979), S. 90-106, hier S. 101.

10 Lebte etwa von 124/3 bis 88/87 v. Chr.

11 Klaus Schippmann, Grundzüge der parthischen Geschichte, Darmstadt 1980, S. 90.

12 Lukas Nickel, The First Emperor and sculpture in China, in: Bulletin of School of Oriental and African Studies 76 (2013), S. 413-447, hier S. 427.

13 Schippmann, Grundzüge, S. 90

14 Nickel, The First Emperor and sculpture in China, S. 423.

15 Josef Wiesehöfer, Das frühe Persien. Geschichte eines antiken Weltreichs, München 2015, S. 83-84. 
Indizien einer strukturellen Instabilität vorliegen. ${ }^{16}$ Auch der Umstand, dass unter den Arsakiden kein stehendes königliches Heer existierte und die Herrscher auf die Unterstützung der Adelsgeschlechter angewiesen waren, kann ob der militärischen Schlagkraft und des langen Bestands des Reichs nicht als Schwäche interpretiert werden. ${ }^{17}$

Die Satrapie Parthava bestand bereits unter den Achaimeniden und Seleukiden und umfasste die Kleinsatrapien Parthien und Hyrkanien. Parthien lässt sich im Becken der Kavir-Wüste im Iran verorten, Hyrkanien befand sich nördlich dieser Provinz. ${ }^{18}$ Während des dritten Syrischen Krieges wurde diese Provinz unter ihrem Satrapen unabhängig. Diese wurde wiederum durch die einfallenden skythischen Parner unter Arsakes, dem Begründer der Dynastie der Arsakiden, eingenommen. ${ }^{19}$ Als die Parner ihre Herrschaft auf weitere Gebiete ausdehnten, nahmen sie die Bezeichnung Parther an. In der Forschung wird angenommen, dass mit der Ernennung von Arsakes zum König die Datierung der Arsakiden beginnt. ${ }^{20}$

Die Parther mussten oft gleichzeitige Bedrohungen aus dem Osten und Westen bewältigen. Die erste derartige uns bekannte Auseinandersetzung erfolgte unter Mithridates $1 .{ }^{21}$ zwischen den Parthern, den Seleukiden im Westen und den Yue-Chi im Osten. Die Bedrohung durch Nomadenvölker im Osten, die sich oft zeigen sollte, wird in den überlieferten Quellen dagegen wenig thematisiert, da diese entweder rom- oder sinozentristisch sind. Dass die Parther dies anders sahen, zeigt sich im konkreten Fall daran, dass Mithridates in dieser Auseinandersetzung im Osten kämpfte und sich im Westen durch einen Feldherrn vertreten ließ.22

In der Regierungszeit von Mithridates I. und Mithridates II. kam es zu großen Ausdehnungen des Partherreichs. Unter Mithridates I. konnten Gebiete im Westiran, Mesopotamien und Teile des Graeco-Baktrischen Reiches eingenommen werden. ${ }^{23}$ Gebietsverluste unter seinem Nachfolger Phraates II. ${ }^{24}$ wurden unter Mithridates II. überwunden. Charakene, Dura Europos, Adiabene, Gordyene und Osrhoene wurden Vasallenstaaten und das Partherreich konnte seinen Einfluss bis nach Armenien ausdehnen. In diese Zeit fiel auch die Gesandtschaft des Kaisers Wu-ti, mit der die Öffnung der Seidenstraße datiert wird.

Auch zu den Römern entstanden in dieser Zeit erste diplomatische Kontakte und die später vielfach umkämpfte Grenze des Euphrat wurde zwischen den beiden Reichen

16 Josef Wiesehöfer, Parther und Sasaniden. Imperien zwischen Rom und China, in: Michael Gehler/Robert Rollinger (Hrsg.), Imperien und Reiche in der Weltgeschichte. Epochenübergreifende und globalhistorische Vergleiche, Bd. 1. Wiesbaden 2014, S. 449-478, hier S. 455-457.

17 Henning Börm, Kontinuität im Wandel. Begründungsmuster und Handlungsspielräume der iranischen Monarchie in arsakidischer und sasanidischer Zeit; in: Stefan Rebenich (Hrsg.), Monarchische Herrschaft im Altertum (Schriften des Historischen Kollegs 94), München 2017, S. 545-564, hier S. 550.

18 Bruno Jacobs, Die Satrapienverwaltung im Perserreich zur Zeit Darius' III. (Beihefte zum Tübinger Atlas des Vorderen Orients B 87), Wiesbaden 1994, S. 187-190.

19 Wiesehöfer, Das frühe Persien, S. 92.

20 Schippmann, Grundzüge, S. 12-17.

21 Regierte etwa von 171 bis 139/38 v. Chr.

22 Schippmann, Grundzüge, S. 23-25.

23 Wiesehöfer, Parther und Sasaniden, S. 450.

24 Regierte etwa von 139/38 bis 128 v. Chr.; Schippmann, Grundzüge, S. 27. 
festgesetzt. ${ }^{25}$ Diese wurde bereits 66 v. Chr. erstmals von den Römern überschritten. Die bekannteste Auseinandersetzung zwischen Römern und Parthern ereignete sich 53 v. Chr. bei Carrhae. Bei dieser Schlacht handelte es sich zwar nicht um den miliärisch verlustreichsten Konflikt zwischen diesen beiden Reichen; trotzdem erhielt sie bei den Römern beinahe traumatische Bedeutung, ${ }^{26}$ die sich auch in späteren römischen Quellen widerspiegelte. Erst unter Augustus kam es, römischen Quellen zufolge, zu einer diplomatischen Lösung: Die römischen Feldzeichen, die in Carrhae erbeutet worden waren, wurden zurückerstattet. ${ }^{27}$ In der Forschungsliteratur wird ein positiver Zusammenhang zwischen dem hierdurch eingeleiteten Frieden und dem Handel entlang der Seidenstraße hergestellt. ${ }^{28}$ Durch eine Krise in China wurde der Handel über das Partherreich von 8 bis 73 n. Chr. aber erneut unterbrochen und verlagerte sich auf den Seeweg über Indien. ${ }^{29}$

Die Herrschaft über Armenien - der Stein des Anstoßes vieler Auseinandersetzungen zwischen Römern und Parthern - blieb umstritten. Erst unter Vologaeses I. ${ }^{30}$ konnte ein faktischer Frieden zwischen den Großmächten geschlossen werden, indem Tiridates, ein Bruder des Vologaeses, in Rom zum armenischen Herrscher gekrönt wurde. Dadurch wurde die tatsächliche Herrschaft eines arsakidischen Herrschers gesichert, aber der faktische Anspruch der Römer auf Armenien durch die Parther anerkannt. ${ }^{31}$

Unter Trajan ${ }^{32}$ kam es zu einem weiteren römischen Feldzug gegen die Parther; im Laufe dessen wurde Adiabene besetzt und Ktesiphon, die parthische Hauptstadt am Tigris, eingenommen. Diese Gebiete wurden unter Hadrian allerdings wieder aufgegeben. Während des zweiten Jahrhunderts n. Chr. kam es zu weiteren Auseinandersetzungen zwischen Rom und den Parthern, im Zuge derer die Römer große Gebietsgewinne machten. Die Parther kämpften in dieser Zeit verstärkt mit dynastischen Problemen. ${ }^{33}$ Das Ende des Partherreichs wurde aber nicht von den Römern, sondern von einer Rebellion im Osten des Reiches ausgelöst. In Persis erhoben sich die Sassaniden; 224 n. Chr. kam es zur Entscheidungsschlacht gegen den sassanidischen Reichsbegründer Ardashir. $^{34}$

\section{Quellen}

\subsection{Parthische Quellen}

Was parthische Quellen selbst anbelangt, ist die Quellensituation schwierig. Archäologisch ist das Partherreich nicht gut erforscht, da bei Ausgrabungen der Fokus auf älteren

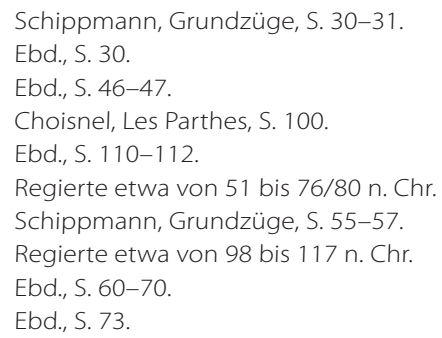


Schichten lag und wichtige parthische Städte noch nicht freigelegt wurden. ${ }^{35}$ Des Weiteren sind die Parther in ihren Provinzen nicht als eigene Entität greifbar, da sie lokale Verwaltungen bestehen ließen und nicht in religiöse oder kulturelle Traditionen eingriffen. ${ }^{36}$ Eine eigene Geschichtsschreibung oder Literatur der Parther sind nicht bekannt. ${ }^{37}$

Die Überlieferung ist in den Regionen des Partherreichs unterschiedlich. Da die parthische Sprache nie verbindliche Reichssprache war, ist der Großteil der auf parthischem Gebiet gefundenen Quellen in regionalen Sprachen abgefasst ${ }^{38}$; daher ist die Überlieferung in Babylonien aufgrund der Verwendung von Tontafeln ${ }^{39}$ besser als in Regionen, in denen Papyrus oder Pergament verwendet wurde. ${ }^{40}$ Vor allem die parthische Numismatik liefert aufschlussreiche Informationen über die parthische Geschichte.

\subsection{Römische Quellen}

Die Parther wurden, als bedeutende Feinde Roms, in römischen und griechischen Quellen stark rezipiert. In der Antike wurden mehrere historiographische Schriften über das Partherreich verfasst, von denen aber keine vollständig überliefert sind. ${ }^{41}$ Grundsätzlich müssen römische Informationen über das Partherreich mit Vorsicht behandelt werden, da es sich um einen Blick von außen handelt und in diesen Quellen auch römische Propaganda mitschwingt. ${ }^{42}$ Zusätzlich werden hier oft Stereotype und negative Topoi verwendet.

Nach Sabine Müller und Josef Wiesehöfer (2017) werden Topoi, die bereits in der griechischen Literatur für das Perserreich verwendet wurden, auf das Partherreich übertragen; es kam zu einer deskriptiven Gleichsetzung beider Reiche. Beispiele solcher Stereotypen sind die Verwendung des Barbarentopos, der Tyrannentopik, der negativen Auswirkung von Dekadenz und diverser negativer Orientbilder, ${ }^{43}$ wie der Schilderung von Haremsintrigen, Blutschande oder der Verweis auf die Wollust der Arsakiden. ${ }^{44}$

Römische Historiographen, wie Tacitus, beschäftigen sich außerdem primär mit den Parthern, wenn sie Konfliktgeschichte schrieben. Der Fokus dieser Autoren lag daher auf dem Kriegswesen der Parther, ihren Königen und ihrer Aristokratie. Themen wie das Alltagsleben im Partherreich oder auch ihre Wirtschafts- und Handelsbeziehungen spielten eine untergeordnete Rolle. Überdies lag der Fokus der römischen Historiographen auf der Westhälfte des Partherreichs. Es kann aber davon ausgegangen wer-

\footnotetext{
35 Wolfram Grajetzki, Greeks and Parthians in Mesopotamia and beyond, 331 BC-224 AD, London 2011, S. 29.

36 Bruno Jacobs, Verwaltung, in: Hackl/Jacobs/Weber (Hrsg.), Quellen, S. 84-100, hier S. 97.

37 Josef Wiesehöfer/Sabine Müller, Einführung, in: Josef Wiesehöfer/Sabine Müller (Hrsg.), Parthika. Greek and Roman Authors'Views of the Arsacid Empire, Wiesbaden 2017, S. VII-XIII, hier S. IX.

38 Hackl, Schriftquellen zur Geschichte des Partherreichs, S. 21.

39 Jacobs, Verwaltung, S. 84.

40 Marek Jan Olbrycht, Greeks in the Arsacid Empire, in: Müler/Wiesehöfer (Hrsg.), Parthika, S. 3-27, hier S. 21

41 Schippmann, Grundzüge, S. 5.

42 Hackl, Das Partherreich und Rom, S. 57.

43 Müller, Das Bild der Parther bei Trogus-Justin, S. 250.

44 Irene Huber/Udo Hartmann, 'Denn ihrem Diktat vermochte der König nicht zu widersprechen'. Die Position der Frauen am Hof der Arsakiden, in: Antonio Panaino/Andrea Piras (Hrsg.), Proceedings of the Fifth Conference of Iranian Studies held in Ravenna, 6-11 October 2003 (Ancient \& Middle Iranian Studies 1), Milano 2006, S. 486-517, hier S. 497-498.
} 
den, dass für die Parther die Osthälfte ihres Reiches und deren Grenze ebenso wichtig waren. ${ }^{45}$

In dieser Arbeit werden die "Naturalis Historia"46 des Plinius, die "Parthischen Stationen"47 des Isidor von Charax und die "Geschichte des Kaisertums nach Marcus"48 von Herodian näher untersucht, da sie im Vergleich zu anderen römischen Quellen wenige negative Topoi aufweisen.

Gaius Plinius Secundus der Ältere ${ }^{49}$ durchlief die römische Beamten- und Militärlaufbahn und verbrachte in diesem Zusammenhang Zeit in Syrien und Judäa. Sein Hauptwerk ist die "Naturalis Historia", die 37 Bücher und verschiedene Wissensbereiche der Natur umfasst sowie als eine Exzerptensammlung beschrieben werden kann. Mit den Gebieten des Partherreichs beschäftigte sich Plinius im sechsten Buch der "Naturalis Historia", in der die Geographie Asiens behandelt wird. ${ }^{50}$ Plinius legte in der Beschreibung des Partherreichs keinen Fokus auf die oben genannten negativen Topoi. Übersetzungen wurden von Kai Brodersen (1998) ${ }^{51}$ und Roderich König/Gerhard Winkler $(1977)^{52}$ durchgeführt.

Isidor von Charax schrieb im ersten Jahrhundert n. Chr. und stammte aus der Stadt Charax im Partherreich. Ob es sich bei dieser Siedlung um die Hauptstadt der Charakene, Charax Spasinou, handelte, ist in der Forschung umstritten. Sollte Isidor aus Charax Spasinou stammen, wuchs er an einem der wichtigsten Handelsumschlagplätze zwischen dem Römischen Reich und Indien auf. Mit großer Wahrscheinlichkeit war er Teil einer hellenistisch geprägten Familie. ${ }^{53}$ Seine Werke verfasste er auf Griechisch. Ein größeres von Isidor verfasstes geographisches Werk ist nicht überliefert; aus seiner Schrift über das Partherreich sind nur Fragmente überliefert, zu denen sowohl die „Parthischen Stationen" als auch ein Fragment über die Perlenfischerei gehören dürften. In den "Parthischen Stationen" wird die Route von Zeugma bis nach Alexandropolis in Arachosien beschrieben. ${ }^{54}$ Das Werk wird in einen griechisch/römischen und nicht in einen parthischen Kontext gesetzt.

Herodian stammte wahrscheinlich aus Syrien und wurde um 180 n. Chr. geboren. Er verfasste eine "Geschichte des Kaisertums nach Marcus", die acht Bücher umfasst. Sie

45 Matthäus Heil, Die Parther bei Tacitus, in: in: Müller/Wiesehöfer (Hrsg.), Parthika, S. 259-278, hier S. 263-264.

46 Gaius Plinius Secundus der Ältere, Naturkunde. Buch VI, lateinisch-deutsch, übersetzt und herausgegeben von Kai Brodersen (Sammlung Tusculum), Zürich 1998; Gaius Plinius Secundus der Ältere, Naturkunde. Bücher XII/XIII, lateinisch-deutsch, übersetzt und herausgegeben von Roderich König/Gerhard Winkler (Sammlung Tusculum), München 1977.

47 Udo Hartmann, Die Parthischen Stationen des Isidor von Charax: eine Handelsroute, eine Militärkarte oder ein Werk geographischer Gelehrsamkeit?, in: in: Müller/Wiesehöfer (Hrsg.), Parthika, S. 87-125.

48 Herodian, Geschichte des Kaisertums nach Marcus, griechisch-deutsch, übersetzt von Lukas Thommen, herausgegeben von Ursula Hackl/Bruno Jacobs/Dieter Weber, in: Ursula Hackl/Bruno Jacobs/ Dieter Weber (Hrsg.), Quellen zur Geschichte des Partherreiches. Textsammlung mit Übersetzungen und Kommentaren, Bd. 2 (Novum Testamentum et Orbis antiquus 84), Göttingen u. a. 2010, S. 174-189.

49 Lebte etwa von 23/24 bis 79 n. Chr.

50 Lukas Thommen, Literarische Texte, in: Hackl/Jacobs/Weber (Hrsg.), Quellen, S. 22-434, hier S. 307-308.

51 Plin. NH. VI.

52 Plin. NH. XII/XIII.

53 Hartmann, Die Parthischen Stationen, S. 89.

54 Thommen, Literarische Texte, S. 190. 
beschreibt die Zeit nach dem Tod Marc Aurels (180 n. Chr.) bis zur Thronbesteigung von Gordian III. (238 n. Chr.). Als Hauptquelle verwendete er Cassius Dio. Auf das Partherreich ging Herodian im Zusammenhang mit dessen Konfliktgeschichte mit dem Römischen Reich ein. Die Beschreibungen des Partherreichs weisen gewisse Stereotype auf. ${ }^{55}$ Generell wird das Werk des Herodian in der Forschung kritisch und als nicht zuverlässig angesehen. ${ }^{56}$ Eine Übersetzung wurde von Lukas Thommen (2010) $)^{57}$ verfasst.

\subsection{Chinesische Quellen}

In dieser Arbeit werden drei chinesische Quellen näher untersucht, in denen die Parther und ihre Beziehungen zu den Han behandelt werden: das „Shiji“58 des Sima Qian, das "Hou Hanshu"59 des Fan Ye und das „Jinshu“60 des Fan Xuanling. Die verwendeten Quellen sind sinozentristisch, daher müssen Informationen über andere Länder und Ethnien kritisch hinterfragt werden. Auf eine Verwendung des "Hanshu“ des Ban Gu wird verzichtet, da hieraus im Vergleich zu dem „Shiji“ keine neuen Informationen über die Parther hervorgehen. Quellen, die nach dem Untergang des Partherreichs entstanden sind, wie das „Hou Hanshu“ und das „Jinshu“, verwendeten oft Textstellen aus früheren historiographischen Schriften, die nur gering umgeschrieben wurden; ${ }^{61}$ daher werden auch diese nicht herangezogen. Eine Übersetzung wurde von Uta Golze und Kerstin Storm (2010) ${ }^{62}$ durchgeführt.

China hat eine lange Tradition der Historiographie. In der Literatur wird das "Shiji“ des Sima Qian vielfach mit dem Werk Herodots verglichen. ${ }^{63}$ Zugeschrieben wird dieses

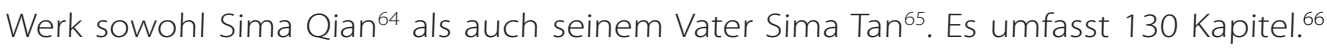
Sima Qian war Diener und Astrologe am Hof des Kaisers Wu; daher muss von einer gewissen Abhängigkeit von diesem Hof ausgegangen werden, die aber nicht überschätzt werden sollte, da er wegen kritischer Bemerkungen am Hof in Ungnade fiel. Derartige kritische Bemerkungen sind auch in seinem Werk zu finden. Das „Shiji“ entstand nicht im Rahmen seiner offiziellen Tätigkeit, sondern als Privatprojekt. ${ }^{67}$

Thommen, Literarische Texte, S. 174.

56 Z. B. Hackl, Handel, S. 120.

57 Herodian, Geschichte des Kaisertums nach Marcus.

58 Sima Qian, Shiji, chinesisch-deutsch, übersetzt von Uta Golze und Kerstin Storm, herausgegeben von Ursula Hackl/Bruno Jacobs/Dieter Weber, in: Ursula Hackl/Bruno Jacobs/ Dieter Weber (Hrsg.), Quellen zur Geschichte des Partherreiches. Textsammlung mit Übersetzungen und Kommentaren, Bd. 3 (Novum Testamentum et Orbis antiquus 85), Göttingen u. a. 2010, S. 487.

59 Fan Ye, Hou Hanshu, übersetzt von Uta Golze und Kerstin Storm, herausgegeben von Ursula Hackl/Bruno Jacobs/ Dieter Weber, in: Hackl/Jacobs/ Weber (Hrsg.), Quellen, Bd. 3, S. 497.

60 Fan Xuanling, Jinshu, übersetzt von Uta Golze und Kerstin Storm, herausgegeben von Ursula Hackl/Bruno Jacobs/ Dieter Weber, in: Hackl/Jacobs/Weber (Hrsg.), Quellen, Bd. 3, S. 502.

61 Uta Golze/Kerstin Storm, Chinesische Quellen zum Partherreich, in: Hackl/Jacobs/Weber (Hrsg.), Quellen, S. 482512, hier S. 484.

62 Sima Qian, Shiji; Fan Ye, Hou Hanshu; Fan Xuanling, Jinshu.

63 Z. B. Siep Stuurman, Herodotus and Sima Qian. History and the Anthropological Turn in Ancient Greece and Han China, in: Journal of World History 19 (2008), Heft 1, S. 1-40.

64 Lebte etwa von 145 bis 86 v. Chr.

65 Lebte bis etwa 110 v. Chr.

66 Golze/Storm, Chinesische Quellen, S. 487.

67 Stuurman, Herodotus and Sima Qian, S. 7. 
Mit dem "Shiji“ verfolgte Sima Qian das Ziel, eine Geschichte der bekannten Welt zu schreiben. Er ging vor allem auf Themen der Ethnographie wie auch der Geographie ein. Zeitlich umspannt das Werk die Geschichte Chinas von seinen mythischen Ursprüngen bis in die Han-Zeit. Als größter Gegner der Chinesen treten hier die Xiongnu auf, ein "nomadischer Volksstamm, der Chinas nördliche und westliche Grenzen bedrohte" ${ }^{\prime 68}$. Interessanterweise werden diese von Sima Qian nicht nur negativ durch die Verwendung von Stereotypen und Barbarentopoi dargestellt. Derartige Darstellungen sind Teil des „Shiji", überwiegen aber nicht, da auch positive Seiten des Gegners beschrieben werden. ${ }^{69}$ Dies lässt darauf schließen, dass auch Beschreibungen der Parther nicht überwiegend durch Stereotype und Topoi geprägt waren. Informationen über das Partherreich im „Shiji“ basieren aber nicht auf Augenzeugenberichten, sondern auf Berichten Dritter. ${ }^{70}$

Da Kapitel 123 des „Shiji“ beinahe wörtlich im „Hanshu“ (Erstes Jahrhundert n. Chr.) des Ban Gu wiedergegeben wird, wird von manchen Wissenschaftler*innen seine Authentizität angezweifelt. Dabei gehen sie davon aus, dass dieses Kapitel später eingefügt worden sein könnte. ${ }^{71}$

Das Hou "Hanshu" wird Fan Ye ${ }^{72}$ zugeschrieben und thematisiert die Regierungszeit der späteren Han von 23 bis 220 n. Chr. ${ }^{73}$ Da das Werk zweihundert Jahre nach dem Ende des Partherreichs entstanden ist, müssen die hier überlieferten Informationen hinterfragt werden. Subjektive Einschätzungen und Wertungen sind im Text des Fan Ye oft durch ein vorangestelltes „in der Diskussion sagen wir" erkennbar und können daher als solche dekonstruiert werden. ${ }^{74}$ Wissenschaftler*innen gehen davon aus, dass Fan Ye nahe an der von ihm verwendeten Vorlage des „Dongguan Hanji“ geblieben ist, das vom ersten bis zum dritten Jahrhundert $n$. Chr. entstand und damit eine zeitgenössische Quelle für das Partherreich war. ${ }^{75}$ Dies ist plausibel, da in der chinesischen Historiographie oft Textstellen von früheren Texten übernommen wurden.

Das „Jinshu“ des Fan Xuanling ist zeitlich noch weiter von den Parthern entfernt, als es das "Hou Hanshu" ist, da es vermutlich nicht vor dem siebten Jahrhundert n. Chr. geschrieben wurde. Die meisten hier überlieferten Informationen basieren auf älteren Texten. ${ }^{76}$

68 Golze/Storm, Chinesische Quellen, S. 482.

69 Stuurman, Herodotus and Sima Qian, S. 2-28.

70 Golze/Storm, Chinesische Quellen, S. 482.

71 Ebd., S. 488

72 Lebte etwa von 398 bis 446 n. Chr.

73 Golze/Storm, Chinesische Quellen, S. 497.

74 Homer H. Dubs, The Reliability of Chinese Histories, in: The Far Eastern Quarterly 6 (1946), Heft 1, S. 23-43, hier S. 30.

75 Golze/Storm, Chinesische Quellen, S. 497.

76 Ebd., S. 502-503. 


\section{Analyse}

Im Folgenden werden die genannten Quellen genauer analysiert. Lassen sich aus ihnen direkt Rückschlüsse auf die Wirtschaft des Partherreichs ziehen, werden sie bereits im folgenden Punkt dargelegt.

\subsection{Wirtschaft im Partherreich}

Um die Rolle der Parther im Rahmen des Handels der Seidenstraße bestimmen zu können, ist es wichtig, die im Partherreich produzierten und gehandelten Waren zu identifizieren. Über die wirtschaftliche Produktion, insbesondere über Landwirtschaft und Handwerk zur Zeit der Parther ist wenig bekannt. Archäologische Funde, wie das Handwerkerviertel in Merv, sind bereits in die seleukidische Zeit zu datieren. ${ }^{77}$ Hackl argumentiert, dass die Parther zu Beginn ihrer Regierungszeit das unter den Seleukiden etablierte Handels- und Wirtschaftskonzept unverändert bestehen ließen. ${ }^{78}$ Malcolm Colledge geht davon aus, dass Landwirtschaft und Handel den wichtigsten Teil der parthischen Wirtschaft ausmachten und die Produktion handwerklicher Erzeugnisse nur eine untergeordnete Rolle spielte. Es wurden Schafe, Ziegen, Rinder, Schweine, Pferde, Esel und Kamele gehalten und Getreide, Reis, Früchte und Gemüse angebaut. In diesem Zusammenhang waren Bewässerungstechniken von Bedeutung, die unter den Parthern weiter ausgebaut wurden. ${ }^{79}$ Der angeblich untergeordneten Bedeutung des Handwerks im Partherreich widersprechen römische Texte, die parthische Metalle, Schmuck und Eisen erwähnen. Aus den Quellen geht auch hervor, dass gewerbsmäßige Perlenfischerei, Bergwerke und Werften für Schiffe bestanden. ${ }^{80}$

In der Literatur wird argumentiert, dass die Parther primär als Mittelsmänner agierten und nicht als Produzenten und Exporteure in den Handel der Seidenstraße eintraten. ${ }^{81}$ Dem widersprechen chinesische Quellen, aus denen hervorgeht, dass Granatäpfel, Pferde aus Nisa, Luzerne und Weinstöcke aus Medien, einer Provinz des Partherreiches, nach China exportiert wurden. ${ }^{82}$ Auch Perlen aus dem Persischen Golf wurden geschätzt. ${ }^{83}$ In zahlreichen Ostraka, die in Nisa, der im heutigen Turkmenistan gelegenen Hauptstadt des Partherreichs, ${ }^{84}$ gefunden wurden und die zu einem großen Teil Weinlieferungen und Abgaben behandeln, zeigt sich die Bedeutung von Wein im Partherreich. ${ }^{85}$ Aus dem „Periplus Maris Erythraiei"86 ergibt sich darüber hinaus, dass Kupfer, verschiedene Edelhölzer, Weihrauch und Seidenstoffe in das Partherreich importiert wurden. Ob diese Waren weiter transportiert wurden oder im Partherreich verblieben, geht aus der Quelle nicht hervor. Seidenstoffe wurden jedenfalls nicht bloß weiter-

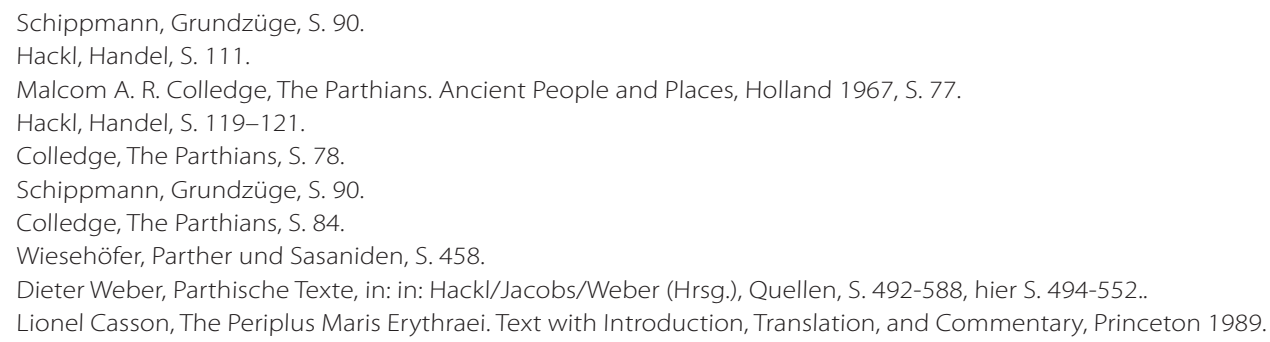


transportiert, da die Parther eine Vorliebe für kostbare Stoffe hatten und es Hinweise auf eine Weiterverarbeitung im Partherreich gibt. Der Import von Edelsteinen wird im sogenannten Perlenlied erwähnt. ${ }^{87}$

\subsection{Steuern und Abgaben}

Über von den Parthern erhobene Steuern und Abgaben ist wenig bekannt. Bestenfalls können Rückschlüsse aus der Zeit der Achaimeniden oder Sassaniden gezogen werden. ${ }^{88}$ Die beste Quellenlage findet sich, aus den bereits beschriebenen Gründen, in Mesopotamien, wo eine Kopf- und eine Grundsteuer bekannt sind. Ostraka zeigen zusätzlich, dass aufWeinberge in Nisa Naturalsteuern erhoben wurden. ${ }^{89}$ Sowohl Hackl als auch Schippmann gehen davon aus, dass die Parther von dem durch ihr Land verlaufenden Handel profitiert haben. Schippman meint, dass die Parther Zölle und verschiedene Steuern, wie sie aus Palmyra und dem römischen Reich bekannt sind, erhoben haben. ${ }^{90}$

\subsection{Handelsmetropolen im Partherreich}

Die Bedeutung von bestimmten Städten als Handelsmetropolen an der Seidenstraße ist schwer nachzuweisen, da es nicht leicht ist, auf Basis von Ausgrabungen zwischen "regulären" und Fernhandelsaktivitäten zu unterscheiden. ${ }^{91}$ Klare Hinweise liefern Inschriften, wie sie zum Beispiel aus Palmyra bekannt sind, das zwar unter römischem Einfluss stand, aber über lange Zeit autonom zwischen Rom und dem Partherreich handeln konnte. Die Bedeutung Palmyras für den Handel der Seidenstraße lag in der Rolle der Palmyrener als Händler zwischen dem Partherreich und Rom. ${ }^{92}$ Als solche waren sie vom Wohlwollen ihrer Handelspartner abhängig, das ihnen von den Parthern nicht immer entgegengebracht wurde. ${ }^{93}$ Sie konnten aber auch in einer gewissen Machtposition auftreten, wie sich in der Stationierung eines palmyrenischen Regiments am mittleren Euphrat im zweiten Jahrhundert n. Chr. zeigt. ${ }^{94}$ Die Inschriften Palmyras geben „Aufschluss zu Aktionsradius und Organisationsform“95 des Karawanenhandels. Für Seleukia am Tigris/Ktesiphon ist beispielsweise eine palmyrenische Inschrift aus dem ersten Jahrhundert n. Chr. belegt, in der sowohl eine Gemeinschaft griechischer als auch palmyrenischer Händler in der Stadt genannt wird. Für Nisibis wurden Aktivitäten von Seidenhändlern nachgewiesen,96 und auch für Babylon ist eine palmyrenische Händlergemeinschaft durch Inschriften aus Palmyra belegt. ${ }^{97}$ Die geographische Lage

\footnotetext{
87 Hackl, Handel, S. 119-120.

88 Ebd., S. 122.

89 Schippmann, Grundzüge, S. 92.

90 Ebd., S. 91

91 Young, Rome's Eastern Trade, S. 11

92 Lisa-Marie Gabriel, Von der Oase zur antiken Handelsmetropole. Überlegungen zur wirtschaftlichen Rolle und Bedeutung der nahöstlichen Oasenstadt Palmyra im Kontext der römischen Ostexpansion, in: historia.scribere 9 (2017), S. 213-242, hier S. 221-223, [http://historia.scribere.at/], eingesehen 5. 4. 2019.

93 Ebd., S. 227

94 Ebd., S. 235.

95 Ebd., S. 225.

96 Young, Romess Eastern Trade, S. 190.

97 Ebd., S. 140
} 
von Städten spielte ebenfalls eine wichtige Rolle; die Lage von Nisa beispielsweise erlaubte die Kontrolle über einen strategisch wichtigen Gebirgspass auf dem Weg in den Iran und damit die Kontrolle dieses Zweigs des Handelswegs. ${ }^{98}$ Eine der wichtigsten Handelsmetropolen war Charax Spasinou, die Hauptstadt der Charakene, da in dieser Stadt Waren aus Indien verladen wurden, die über den Seeweg gehandelt wurden. ${ }^{99}$ Bei Mesene handelte es sich um einen Vasallen des Partherreichs, der stark unter palmyrenischem Einfluss stand und im zweiten Jahrhundert n. Chr. kurzzeitig römischer Vasall wurde. ${ }^{100}$ Die Stadt Zeugma ist in diesem Zusammenhang einerseits durch einen wichtigen Übergang über den Euphrat, ${ }^{101}$ andererseits durch einen jährlichen Markt bekannt, auf dem chinesische und indische Waren verkauft wurden. ${ }^{102}$

Weitere Hinweise auf wichtige Handelspunkte liefern die „Parthischen Stationen" des Isidor von Charax. Die Bedeutung und Intention dieses Werks ist in der Forschung jedoch stark umstritten. Die neuere Forschung geht nicht mehr davon aus, dass es sich bei diesem Werk um eine Beschreibung von Handelswegen handelt, sondern vielmehr um Militärrouten. ${ }^{103}$ Auch diese Deutung ist allerdings umstritten, da die in den „parthischen Stationen“ angeführten Informationen für militärische Zwecke nur bedingt brauchbar sind. ${ }^{104}$ Indes, auch wenn bei den "Stathmoi Parthikoi“ davon ausgegangen werden muss, dass es sich nicht primär um Handelsstationen handelte, sind diese trotzdem interessant, da es sich nach Hartmann um „vom Staat unterhaltene Versorgungsstützpunkte handelte, die das sichere und schnelle Reisen durch das Großreich ermöglichen sollten"105. Auch wenn keine konkreten Hinweise auf eine Nutzung der Strecke für den Fernhandel nach China und Indien vorliegen, kann eine derartige Nutzung nicht vollständig ausgeschlossen werden. ${ }^{106}$ Zusätzlich gibt es Überschneidungen zwischen einigen der in den "Stathmoi“ genannten Orten mit aus anderen Quellen bekannten Handelsmetropolen: Zeugma, Seleukia am Tigris, Charax Spasinou, etc. ${ }^{107}$

\subsection{Numismatik}

Rückschlüsse auf die Entwicklung der Wirtschaft und den Wohlstand im Partherreich lassen sich unter anderem auf Basis der formalen Ausbildung, der Fundmenge und des Silbergehalts der dort geprägten Münzen ziehen. Interpretationen auf Basis der Fundmenge dürfen allerdings nicht überbewertet werden, da diese zu einem großen Teil von der Zufälligkeit archäologischer Funde beeinflusst sind. Hier soll nur ein klei-

\footnotetext{
98 Choisnel, Les Parthes, S. 30.

99 Young, Rome's Eastern Trade, S. 92.

100 Ebd., S. 146.

101 Ebd., S. 188-189.

102 Vladimir Grigor'evich Lukonin, Political, Social and Administrative Institutions. Taxes and Trade, in: Insān Yāršātir (Hrsg.), The Cambridge history of Iran, Bd. 3/2, Cambridge 1983, S. 681-746, hier S. 740.

103 Hackl, Handel, S. 112.

104 Hartmann, Die Parthischen Stationen, S. 109-110.

105 Ebd., S. 103.

106 Ebd., S. 107

107 Monika Schuol, Isidor von Charax und die literarische Gattung der Stathmoi, in: Müller/Wiesehöfer (Hrsg.), Parthika, S. 71-85, hier S. 71.
} 
ner Überblick zu diesem Thema gegeben werden, um die Auswirkungen von Unterbrechungen oder Verschiebungen des Handels aufgrund militärischer Auseinandersetzungen oder politischer Verlagerungen zu beleuchten. ${ }^{108}$ Es muss vorangestellt werden, dass bei einem Vergleich der Entwicklung des Silbergehalts parthischer und römischer Münzen generell eine Entwertung über einen längeren Zeitraum zu beobachten ist. ${ }^{109}$

Größere Entwertungen sind unter Orodes II. ${ }^{110}$ belegt, in dessen Regierungszeit die Schlacht von Carrhae 53 v. Chr. fiel. Diese Entwertung wird in der Forschungsliteratur auf zwei Gründe zurückgeführt. Einerseits wird sie durch größere Ausgaben aufgrund militärischer Auseinandersetzungen mit dem Römischen Reich und innerdynastische Streitigkeiten erklärt. Andererseits wird eine Verschlechterung der Wirtschaft im Partherreich selbst angenommen, die sich aus einer Verlegung der Handelsrouten der Seidenstraße ergeben haben soll. Es wird davon ausgegangen, dass der Handel in dieser Zeit über Wege außerhalb des Partherreichs führte, da der Karawanenverkehr durch die militärischen Konflikte nicht mehr sicher war. ${ }^{111}$ Diese Entwicklung setzte sich unter Phraates IV. fort. ${ }^{112}$ Welcher dieser Gründe tatsächlich überwog, kann nicht mehr festgestellt werden. Ebenso können die Folgen weiterer Unterbrechungen des Handels der Seidenstraße, wie sie im ersten Jahrhundert n. Chr. bekannt sind, auf Basis der Untersuchungen von Earle R. Caley ${ }^{113}$ nicht abgeleitet werden. Nach dem ersten Jahrhundert n. Chr. und unter der Friedenspolitik Hadrians scheint der Silbergehalt der untersuchten Tetradrachmen wieder gestiegen zu sein. E. J. Keall stellt fest, dass die Anzahl der unter Vologaeses II. geprägten Münzen hoch war. ${ }^{114}$ Diese Ergebnisse lassen darauf schließen, dass militärische Auseinandersetzungen größere Auswirkungen auf die Wirtschaft der Parther hatten als die Unterbrechung der Landroute der Seidenstraße durch China.

Zudem lassen sich Rückschlüsse auf den Handel aufgrund von Fundorten bzw. Quellen ziehen. Wie das „Shiji“ zeigt, waren den Han-Chinesen parthische Münzen bekannt. Die Beschreibung dieser Münzen macht ein Drittel der zitierten Quellenstellen aus, nimmt also eine verhältnismäßig große Rolle ein. Das Thema der parthischen Münzen wird auch im „Hanshu“115 behandelt; hier wird sogar zusätzlich zur Vorderseite der Münzen deren Rückseite beschrieben. ${ }^{116}$ Die Detailliertheit der Beschreibung legt nahe, dass

108 Für eine detailliertere Untersuchung siehe: David Sellwood, Parthian Coins, in: Iḥān Yāršātir (Hrsg.), The Cambridge history of Iran, Bd. 3/1, Cambridge 1983, S. 279-299; Earle R. Caley, Chemical Composition of Parthian Coins, in: Numismatic Notes and Monographs 129 (1955), S. 1-104; Ein Überblick über die neuere Forschung findet sich bei: Peter Franz Mittag, Bactria and Parthia, in: Michel Amandry/Donal Bateson (Hrsg.), A Survey of Numismatic Research 2002-2007, Glasgow 2009, S. 111-116.

109 Caley, Chemical Composition of Parthian Coins, S. 34.

110 Regierte etwa von 58/57 bis 38 v. Chr.

111 Edward John Keall, Parthian Nippur and Vologases' Southern Strategy. A Hypothesis, in: Journal of the American Oriental Society 95 (1975), Heft 4, S. 620-632, hier S. 621-622.

112 Sellwood, Parthian Coins, S. 290.

113 Caley, Chemical Composition of Parthian Coins, S. 1-104

114 Keall, Parthian Nippur and Vologases'Southern Strategy, S. 630-631.

115 HS 96A, S. 3889-3890.

116 Es wird hier allerdings auf Punkt 4.3 verwiesen - die Echtheit des Kapitel 123 des Shiji wird in der Forschung diskutiert. Dies trifft allerdings nicht auf das Hanshu zu, daher muss der oben getroffene Schluss nicht hinterfragt werden 
dem Autor die Münzen tatsächlich vorlagen und er nicht nur eine Erzählung Reisender wiedergab. Diese Münzen können nun einerseits durch Reisende oder aber durch Handel in das Han-Reich gelangt sein. Dies kann zwar nicht mit Sicherheit bestätigt werden, aber dennoch als Hinweis auf den Handel zwischen diesen beiden Reichen gesehen werden.

Was parthische Münzfunde auf dem Gebiet der Seidenstraße und in China betrifft, sind in der Literatur nur spärlich Informationen zu finden: Zwei Goldmünzen des Mithridates II., die nahe von Yarkand, das auf der südlichen Route der Seidenstraße gelegen ist, gefunden wurden, werden von Choisnel als Hinweis für den Handel auf dieser Route gesehen. ${ }^{117}$ Münzen aus Charakene aus dem ersten Jahrhundert v. Chr. und dem ersten bis zweiten Jahrhundert n. Chr. wurden auf der Insel Failaka, den kuwaitischen Inseln al-'Akaz und Umm an-Namal und in dem Gebiet der heutigen Vereinigten Arabischen Emirate gefunden. Auch dies wird als Zeichen des transkontinentalen Handels gedeutet. ${ }^{118}$

\subsection{Analyse römischer Quellen}

Die ältere Forschungsliteratur verfolgte die These, dass die Beziehungen und auch die Auseinandersetzungen der Römer mit den Parthern, die vordergründig in den römischen Quellen behandelt werden, durch ökonomische Beweggründe motiviert waren. ${ }^{119}$ Den Parthern wurde ein Monopol im Handel mit Seide und anderen Luxusgütern unterstellt, aus dem sie beträchtliche Gewinne erzielt haben sollen. ${ }^{120}$ Auf dieses Handelsmonopol sollen die Römer durch gezielte Interventionen reagiert haben, um die Parther als Intermediär im Handel mit dem Osten auszuschalten. Diese Thesen sind in der neueren Forschung umstritten. ${ }^{121}$

\subsubsection{Plinius}

Der Handel mit dem Osten wird in den römischen Quellen negativ gesehen und mit Genusssucht und Zügellosigkeit assoziiert. Im folgenden Zitat werden die Parther zwar nicht explizit genannt, wohl aber der Umfang des Handels entlang der Seidenstraße beziffert, mit dem die Parther in Bezug auf den Handel mit den Serern (Han-China) in Verbindung gebracht werden können.

"Gewiß aber noch glücklicher ist das Meer Arabiens: denn es stiftet uns die Perlen, und nach den niedrigsten Schätzungen rauben Indien, die Serer und jene Halbinsel unserem Reiche alle Jahre 100 Millionen Sesterzen: soviel kosten uns Luxus und Frauen!"122

Ob es sich bei den genannten „100 Millionen Sesterzen“ um den Preis der Waren selbst

\footnotetext{
117 Choisnel, Les Parthes, S. 60.

118 Grajetzki, Greeks and Parthians, S. 19-20.

119 Young, Rome's Eastern Trade, S. 1.

120 Für dieses Bild siehe primär Punkt 5.3.

121 Z. B. Young, Rome's Eastern Trade, S. 195-200

122 Plin. NH XII,84.
} 
oder um das Handelsbilanzdefizit Roms handelte, ist nicht bekannt. ${ }^{123}$ Auch an der tatsächlichen Höhe dieser Ausgaben kann gezweifelt werden, es handelt sich wohl um einen Schätzwert. Es ist allerdings davon auszugehen, dass Plinius hier auf die Kosten der Waren im Römischen Reich eingeht und nicht auf die Einnahmen, die in anderen Ländern aus diesem Handel entstanden. In diesem Fall hätte er Informationen über die Einnahmen aus einem dezentralen Handel aus drei verschiedenen Reichen haben müssen. Vollkommen ausgeschlossen kann dies allerdings nicht werden.

Es ist plausibel anzunehmen, dass ein Teil der „100 Millionen Sesterzen“ bei den Händlern dieser Waren verblieb. ${ }^{124}$ Ob unter diesen Händlern auch parthische Kaufleute waren, kann aus den Quellen nicht abgeleitet werden; ein großer Prozentsatz des Handels über das Partherreich wurde durch Händler aus Palmyra abgewickelt. ${ }^{25}$ Ein Teil dieser Ausgaben wurde wohl in Form von Steuern und Abgaben in Ländern wie dem Partherreich einbehalten, durch die der Handel verlief. Wie weiter oben beschrieben, fehlen uns allerdings genauere Informationen. Dass es auch innerhalb des Römischen Reichs nochmals zu Aufschlägen auf den Preis der Waren kam, zeigt das folgende Zitat.

„Die Sache verdient es, da Indien unserem Reich in keinem Jahr weniger als 50.000.000 Sesterzen entzieht und Waren dafür gibt, die freilich bei uns für das Hundertfache verkauft werden." ${ }^{\prime \prime 26}$

Die hier beschriebene Preissteigerung ist indes nicht nur auf Steuern und Abgaben, sondern auch auf eine Wieder- oder Weiterverarbeitung der gehandelten Waren innerhalb des Römischen Reichs zurückzuführen. ${ }^{127}$ Diese Weiterverarbeitung kann als eine Erklärung dafür gesehen werden, warum eindeutig aus China stammende Seidenstoffe im Römischen Reich erst ab dem ersten bis dritten Jahrhundert $n$. Chr. identifiziert werden können. ${ }^{128}$ Es ist daher nicht zwingend anzunehmen, dass früher gehandelte Seidenstoffe in Ländern wie dem Partherreich, die auf der Handelsroute lagen, verblieben, selbst wenn dies möglicherweise der Fall war. Die Verwendung von Seide auf parthischen Bannern in der Schlacht von Carrhae 53 v. Chr. ${ }^{129}$ und die Vorliebe der Parther für schöne Stoffe sind aus den Quellen bekannt. 
Plinius zählt, wie das folgende Zitat zeigt, die Parther allerdings nicht zu den vom Handel profitierenden Intermediären, sondern sieht sie mit ähnlichen Problemen wie jene der römischen Wirtschaft konfrontiert.

„Im Allgemeinen aber sind diese Stämme [Anm.: diverse Araberstämme werden in VI 32 beschrieben; Plinius identifiziert die am Handel Beteiligten aber nicht explizit] sehr reich, da bei ihnen die größten Schätze der Römer und Parther verbleiben, weil sie das, was sie aus dem Meer oder den Wäldern nehmen, verkaufen, ohne ihrerseits wieder etwas einzukaufen." ${ }^{130}$

Dies lässt darauf schließen, dass die Parther von den Römern nicht als wesentliche Profiteure des Handels der Seidenstraße angesehen wurden. Militärische Aktionen zur Aufhebung einer angenommenen Vormachtstellung der Parther durch die Römer erscheinen daher nach dieser Quelle unwahrscheinlich.

\subsubsection{Herodian}

Eine der wenigen römischen Historiographen, die explizit auf den Handel der Römer mit den Parthern eingeht, ist Herodian. Wie bereits weiter oben erwähnt, wird er in der Forschung allerdings kritisch gesehen; während Choisnel ihn als glaubwürdige Quelle behandelt, spricht Hackl dem folgenden Zitat jede Glaubwürdigkeit ab. Diese Zeilen sind in den Kontext eines Heiratsgesuchs des Kaisers Caracallas an Artabanos eingebettet.

„Denn alle übrigen Barbarenvölker, die jetzt an die Imperien der Römer und der Parther angrenzten, seien leicht für sie [Anm.: die Parther und Römer] zu erobern, da sie als Einzel-Völker und Bündnisse regiert würden. Aufseiten der Römer stehe ein Heer mit Fußsoldaten, das im Nahkampf mit (Schwert und) Lanzen unbesieglich sei, aufseiten der Parther aber sei eine starke Reiterei und die Erfahrung zielsicherer Bogenschützen. Wenn diese sich vereinigten und in allem Militärischen zusammenarbeiteten, könnten sie leicht die gesamte bewohnte Welt unter einer einzigen Krone beherrschen. Ferner die bei jenen wachsenden Gewürzpflanzen und ihre bewundernswerten Textilien einerseits und andererseits die im Römerreich gerühmten Metalle und ihre hohe Verarbeitungskunst müssen nicht mehr mühsam, selten und heimlich von Händlern über die Grenzen befördert werden, sondern würden, wenn es nur eineinziges (gemeinsames) Land und eine einzige Macht gäbe, beiden gemeinsam und uneingeschränkt zum Nutzen bereitstehen."131

Hält man dieses Zitat für glaubwürdig, findet sich in dieser Beschreibung eines möglichen Bündnisses der Römer mit den Parthern ein Hinweis auf eine momentan bestehende, aktive Handelspolitik: Es wird darauf verwiesen, dass der freie Handel über die Grenzen der genannten Reiche aktuell verhindert werde. Das Argument, dass Waren heimlich über die Grenzen befördert wurden, findet sich allerdings nur in diesem Zitat 
und das Bestehen einer aktiven Handelspolitik im Zusammenhang mit dem Fernhandel wird in der Literatur kritisch gesehen. ${ }^{132}$

Die Ursache des heimlichen Transports in militärischen Auseinandersetzungen zu suchen, die öfter zu Unterbrechungen des Handels führten, ${ }^{133}$ scheint aber auch nicht schlüssig, da hier eine Verlagerung der Handelsrouten wahrscheinlicher als ein heimlicher Transport entlang den unterbrochenen Routen wäre. Auch das Ziel einer Umgehung von staatlichen Tarifen und Steuern durch den heimlichen Import von Waren ist unwahrscheinlich. Die römischen Steuern auf den Import von Waren des Fernhandels sind gut belegt. ${ }^{134}$ Da das oben angeführte Zitat Kaiser Caracalla in den Mund gelegt wird, ist davon auszugehen, dass eine Umgehung von staatlichen Taxen kritischer thematisiert werden würde.

Möglich wäre im Fall des Imports von „im Römerreich gerühmten Metallen“ eine Einschränkung des Handels mit „margianschem Eisen“ auf Seiten der Parther, da der Besitz dieses Metalls einen militärischen Vorteil bringen könnte. Da Herodian allerdings Ende des zweiten Jahrhunderts n. Chr. schreibt, der Handel zu dieser Zeit schon lange bestand und auch sonst keine Hinweise auf derartige Handelsembargos bestehen, muss diese These in das Reich der Spekulation verwiesen werden. Auch die Unterbrechung der Routen über das Partherreich, die im ersten Jahrhundert n. Chr. bestand, war zur Zeit Herodians wieder aufgehoben. Die Behinderung des Handels zwischen den Römern und Parthern wird aber nicht nur von römischen Quellen thematisiert, wie die Analyse chinesischer Quellen im nächsten Kapitel zeigen wird.

\subsection{Analyse chinesischer Quellen}

In den folgenden Zitaten wird das Land Anxi genauer beschrieben, bei dem es sich um das Partherreich handelt. Diese Bezeichnung wird in der Literatur auf Arsak, beziehungsweise das Reich der Arsakiden zurückgeführt. ${ }^{135}$ Eine mögliche Übereinstimmung chinesischer und lateinischer Namen für bestimmte Regionen wird in der Forschung diskutiert, doch bezüglich der Identifikation Anxis als Partherreich herrscht allgemeiner Konsens.

\subsubsection{Sima Qian}

„Anxi liegt wohl einige tausend li westlich von Da Yuezhi. Es ist Sitte, dass die Einheimischen die Felder bestellen, Reis, Weizen und Traubenwein anbauen. Sie haben befestigte Städte wie in Da Yuan. Zu diesem [Reich] gehören einige hundert große und kleine Städte. Das Gebiet ist einige tausend li groß. Es zählt zu den größten Ländern. Es grenzt an den Gui-Fluss. Es gibt Handelsplätze. Einige Einwohner betätigen sich als Kaufleute und sind mit Wagen und Schiffen unterwegs. Sie reisen einige tausend li in benachbarte Länder. Aus Silber stel- 
len sie Münzgeld her. Dieses Münzgeld trägt auf der Vorderseite das Bild des Königs. Wenn der König stirbt, wird das Münzgeld wieder geändert und das Bild des [neuen] Königs erscheint dort. Sie beschreiben Leder mit quer laufenden Linien und verwenden dies als schriftliche Dokumente. Westlich liegt Tiaozhi, im Norden liegen Yancai und Ligan."136

Im „Shji“ werden agrarische Erzeugnisse des Partherreichs beschrieben, die Rückschlüsse auf dessen Wirtschaft zulassen. Auch Münzen und von den Parthern verwendetes Schreibmaterial werden thematisiert. Besonders interessant ist die Erwähnung von Kaufleuten, die mit „Wagen und Schiffen“ unterwegs sind und weite Strecken bereisen. Dies lässt darauf schließen, dass die Parther nicht nur Handel über die Landroute, d. h. mit klassischen Karawanen, sondern auch Handel zur See trieben und zumindest Teile der Bevölkerung aktiv am Handelsgeschehen beteiligt waren. Im Zitat wird auBerdem auf Handel über weite Strecken verwiesen. (Ein chinesisches li beschreibt einen Abstand von 415/416 m; es kann aber davon ausgegangen werden, dass hier ein persisches Abstandsmaß zitiert wird.) ${ }^{137}$ Zumindest aus dieser Quelle geht daher nicht hervor, dass, wie Hackel argumentiert, die Parther nicht der bestimmende Einfluss auf den Handel in ihrem Reich waren, ${ }^{138}$ da das "Shiji" sowohl auf parthische Kaufleute als auch auf ihre Teilnahme am Fernhandel verweist.

\subsubsection{Fan Ye}

Das folgende Zitat behandelt das Handelsdreieck zwischen Han-China, dem Partherreich und dem römischen Reich. Da Qin wird in der Literatur entweder mit dem römischen Orient, also Ägypten, oder dem gesamten römischen Reich, Tianzhu mit Nordwestindien gleichgesetzt. ${ }^{139}$

„[Da Qin] handelt mit Anxi und Tianzhu auf dem Seeweg. Der Gewinn beträgt das Zehnfache. Die Einwohner haben einen aufrechten Charakter, auf dem Markt gibt es keine zwei Preise, Getreide und andere Nahrungsmittel sind immer preiswert. Das Land nutzt seinen Reichtum und seine Überschüsse. Wenn die Boten der Nachbarländer ihre Grenzen erreichen, kommen sie zuerst zu den Poststationen und erreichen dann die Hauptstadt. Dort angekommen, überreichen sie Goldmünzen. Der König [von Da Qin] wollte immer Gesandte zu den Han schicken, aber Anxi wollte mit den Han buntgewebte Seidenstoffe handeln. Aus diesem Grund versperrten sie den Weg und es gelang [Da Qin] nicht, selbst dorthin zu gelangen." ${ }^{140}$

Wenn in der Literatur von einem Handelsmonopol der Parther im Rahmen des Seidenhandels die Rede ist, wird vielfach auf das oben angeführte Zitat verwiesen. Einerseits deutet dieses Zitat auf eine strikte Kontrolle der Handelswege im Partherreich selbst

136 Sima Qian, Shiji, 123,3162.

137 Golze/Storm, Chinesische Quellen, S. 488.

138 Hackl, Handel, S. 118-119.

139 Golze/Storm, Chinesische Quellen, S. 501.

140 Fan Ye, Hou Hanshu, 88,2919. 
hin, da den Römern, nach dieser Quelle, dieser Weg versperrt wurde und von ihnen kein direkter Kontakt mit Han China etabliert werden konnte. Andererseits wird als Grund für diese Kontrolle der Handel mit buntgewebten Seidenstoffen gemeinsam mit einem beträchtlichen Gewinnpotential - "der Gewinn beträgt das Zehnfache" durch den Fernhandel angeführt. Hier werden die Parther also, wie bereits im „Shiji”, als aktive Akteure im Fernhandel beschrieben. Es kann davon ausgegangen werden, dass die genannten Schritte nicht durch einzelne Kaufleute umgesetzt werden konnten, sondern staatliche Strukturen bestehen mussten, die den Transit durch das Partherreich unterbinden konnten. Dieses Zitat erinnert an die weiter oben angeführte Herodian-Textstelle, da eine solche Kontrolle potentiell der Grund für "heimlichen Handel" sein könnte. Ein in der Realität existierendes Monopol im Seidenhandel wird allerdings in der Forschungsliteratur vielfach verneint. Einerseits war China nicht der einzige Produzent von Seide; sowohl die Inder, mit denen die Römer direkt in Handelskontakt standen, als auch die griechische Insel Kos produzierten Seide. Eine Unterscheidung der gefundenen Stoffe nach ihrem Herkunftsort stellt sich als schwierig heraus. ${ }^{141}$ Daher kann nicht eindeutig festgestellt werden, ob Stoffe aus China, die über das Partherreich gehandelt wurden, im Fernhandel überwogen. Andererseits ist davon auszugehen, dass chinesische Seide auch über Seerouten von Indien in das Römische Reich transportiert wurde, der parthische Intermediär also umgangen werden konnte.

Wenn eine derartige Sperre des Landweges tatsächlich bestand, ist nach Young allerdings davon auszugehen, dass diese nicht zum Zweck der Etablierung eines Handelsmonopols errichtet wurde. Werden die Beziehungen Roms mit anderen Ländern analysiert, so liegt nahe, dass militärische Bündnisse für römische Gesandte interessanter als Handelsbündnisse waren. Der Wunsch der Parther, derartige Kontakte zu unterbinden, ist nachvollziehbar. ${ }^{142}$ Eine vollkommene Sperre der Landroute ist außerdem nicht realistisch, da mindestens ein Fall eines Römers, Maes Titianus, bekannt ist, der in der römischen Kaiserzeit durch das Partherreich nach Zentralasien reiste. ${ }^{143}$ Die folgende Textstelle unterstreicht ebenfalls das Bild der Parther als aktive Akteure im Handel. Im Zusammenhang mit dieser Textstelle muss allerdings auf die literarischen Elemente der Beschreibung verwiesen werden, die zur Vorsicht vor einer zu wörtlichen Interpretation mahnen.

„Das Land Anxi liegt bei der Stadt Hedu, von Luoyang ist es 25000 li entfernt. Im Norden grenzt es an Kangju, im Süden grenzt es an Wuyishanli. Das Land umfasst einige tausend li, in einer kleinen Stadt leben einige hundert Familien, die das Militärische regeln. Es handelt sich um das reichste und üppigste Land. An der östlichen Grenze liegt die Stadt Mulu, die auch den Namen Klein-Anxi trägt. Von Luoyang ist sie 20000 li entfernt. Im ersten Jahr der Regierungsperiode zhanghe (87 n. Chr.) des Kaisers Zhang (57-89 n. Chr.) sandte man eine Delegation, die Löwen und Fuba als Tribut überreichte. Das Fuba ist der Gestalt

141 Hansen, The Silk Road, S. 19-20.

142 Young, Rome's Eastern Trade, S. 196.

143 Ebd., S. 190 
nach ein Hirsch, aber es hat keine Hörner. Im 9. Jahr der Regierungsperiode yongyuan (97 n. Chr.) des Kaisers He (80-106 n. Chr.) sandte Generalgouverneur Ban Chao Gan Ying als Boten nach Da Qin. Als der Tiaozhi erreichte, kam er an das große Meer und wollte es überqueren, doch die Schiffer an der westlichen Grenze Anxis sprachen zu Ying: ,Das Wasser des Meeres ist weit. Die, die hin und zurück wollen, brauchen guten Wind und dann [dauert es] drei Monate bevor sie die Überquerung schaffen. Wenn sie auf schwachen Wind stoßen, benötigen sie jedoch zwei Jahre. Daher versorgen sie sich mit Lebensmitteln für drei Jahre, bevor sie in See stechen. Auf See bekommen die Menschen starkes Heimweh und fühlen Sehnsucht, einige unter ihnen sterben.' Als Ying das hörte, hielt er ein. Im 13. Jahr [101 n. Chr.] überbrachte der König von Anxi, Manqu, wiederum Löwen und riesige Vögel aus Tiaozhi als Tribut. Zu dieser Zeit nannte man sie Vögel aus Anxi."144

Hier wird beschrieben, dass auch chinesische Gesandte davon abgehalten wurden, direkt mit Rom in Kontakt zu treten. Im Gegensatz zu den Römern durften chinesische Gesandte, nach diesem Zitat, aber zumindest das Partherreich durchqueren. Dies lässt auf eine differenzierte Wahrnehmung von Rom und Han-China durch die Parther schließen. Folgt man diesem und dem vorhergehenden Zitat aus dem "Hou Hanshu“, ist davon auszugehen, dass die Römer, obwohl sie wichtige Handelspartner waren, primär als militärische Rivalen wahrgenommen wurden, denen eine unbeschränkte Durchquerung des Partherreichs nicht gestattet werden konnte, während Han-China freien Zugang zu diesem Gebiet hatte. An der westlichen Grenze des Partherreichs angekommen, wurde der chinesische Gesandte aber, wie es scheint, durch eine List parthischer Schiffer davon abgehalten, weiterzureisen. Young berichtet, dass davon ausgegangen werden kann, dass der kürzere Landweg, der ebenso in das Römische Reich führte, von den Parthern bewusst verschwiegen wurde, um den direkten Kontakt zwischen den beiden Ländern zu unterbinden. Auch der Seeweg wurde als besonders dramatisch und lang beschrieben, um dieses Ziel zu erreichen. ${ }^{145}$

Im Zusammenhang mit Han-China kann also davon ausgegangen werden, dass für die Parther wirtschaftliche Überlegungen überwogen. Da keine militärischen Auseinandersetzungen zwischen Han-China und dem Partherreich bekannt sind, ergibt dies durchaus Sinn. In der Forschung wird aber auch diskutiert, inwiefern die Unterbindung des römisch-chinesischen Kontakts durch die Parther als realistisch angesehen werden kann, wenn die chinesischen Gesandten bis an die Grenzen des Partherreichs vordringen durften. War eine Unterbindung dieses Kontakts ein primäres Ziel, wäre ein Verbot der Durchquerung des Partherreichs zielführender gewesen, ${ }^{146}$ als auf die Überredungskünste parthischer Schiffer an den Grenzen des Reiches zu vertrauen.

Um bewerten zu können, inwiefern die Beschreibung der von den Schiffern dargelegten Reisedauer übertrieben ist, ist eine Identifikation von Tiaozhi und dem "gro-

144 Fan Ye, Hou Hanshu, 88,2918-2919.

145 Young, Rome's Eastern Trade, S. 196.

146 Ebd., S. 197. 
Ben Meer" notwendig. Wie bereits weiter oben erwähnt, sind Identifikationen der in den chinesischen Quellen verwendeten Bezeichnungen in der Forschung aber nicht eindeutig geklärt. Golze und Storm nennen einige der gängigsten Identifikationen. Mit dem "großen Meer" kann der indische Ozean, der Persische Golf, aber auch das Kaspische Meer, das von zeitgenössischen Historiographen auch mit dem Schwarzen Meer oder dem Aralsee gleichgesetzt wurde, ${ }^{147}$ gemeint sein. Die Mehrheit der Forscher*innen tendiert zu einer Identifikation des Tiaozhi als dem Indischen Ozean. Trifft dies zu, stellt sich die Frage, ob zur Zeit dieser Gesandtschaft die Verwendung der Monsunwinde bereits bekannt war, von den Schiffern also eine bewusst übertriebene Reisedauer angegeben wurde. In der Forschung ist der Beginn der Verwendung der Monsunwinde durch Araber, Griechen und Römer umstritten. Es kann aber davon ausgegangen werden, dass diese zumindest zur Zeit der Niederschrift des „Periplus Maris Erythraei" im ersten Jahrhundert n. Chr. bereits bekannt war. ${ }^{148}$ Auch die Erwähnung des gesteigerten Handels zwischen Indien und Rom bei Strabon (erstes Jahrhundert v. Chr. bis erstes Jahrhundert n. Chr.) lässt auf eine Kenntnis der Monsunwinde schlieBen. ${ }^{149}$ Vergleicht man die im Zitat genannte Mindestreisedauer von drei Monaten mit der in einem Plinius-Zitat genannten Wegzeit von Alexandrien nach Indien ${ }^{150}$, scheint die angeführte Mindestreisedauer nicht übermäßig übertrieben zu sein. ${ }^{151}$ Wird von einer Identifikation des "großen Meeres" als Kaspisches oder Schwarzes Meer ausgegangen, hätte die Strecke schneller zurückgelegt werden können. Die Ausweitung der Reisedauer auf zwei Jahre ist jedenfalls exorbitant.

Das folgende Zitat aus dem „Jinshu“ unterstreicht die Wahrnehmung der Gewinne des Partherreichs aus dem Fernhandel durch China:

„Die Menschen von Anxi und Tianzhu treiben mit [Da Qin] Handel übers Meer, ihr Gewinn beträgt ein Hundertfaches."152

Im Gegensatz zu dem weiter oben angeführten Plinius-Zitat sahen die Chinesen die Parther demzufolge als Nutznießer aus dem Handel, die einiges zu den erwirtschafteten Preisen beitrugen. Die Steigerung der berichteten Gewinne lässt sich auf die literarische Übertreibung in dieser späteren Quelle zurückführen, könnte aber auch die Verhältnisse unter den Sassaniden widerspiegeln, unter denen der Handel entlang der Seidenstraße größere Ausmaße angenommen hatte.

\section{Fazit und Ausblick}

Wie die Analyse der Quellen aufzeigt, unterscheiden sich die römischen und chinesischen Quellen stark. Während römische Quellen nur sekundär auf wirtschaftliche Themen eingehen, wenn es um die Parther geht, nimmt dieses Thema in chinesischen

\footnotetext{
147 Golze/Storm, Chinesische Quellen, S. 490-491.

148 Young, Rome's Eastern Trade, S. 20.

149 Ebd.

150 Plin. NHVI, 101-106.

151 Choisnel, Les Parthes, S. 124

152 Fan Xuanling, Jinshu, 97,2544.
} 
Quellen eine wichtige Rolle ein. Der Umfang der vorhandenen Informationen ist bei den Römern um einiges größer als bei den Chinesen. Die Existenz von Texten aus dem Römischen Reich, die sich primär mit den Parthern, ihrer Geschichte und Gesellschaft beschäftigten, ist uns bekannt, leider wurden diese Texte aber nicht überliefert. Es ist daher möglich, dass auch die Literatur des Römischen Reiches sich mit den Parthern in einem wirtschaftlichen Kontext befasste. Grundsätzlich lässt die Fülle an Informationen über die Parther in römischen Quellen auf eine größere Beschäftigung mit den Parthern schließen, als es für China der Fall war. Da das römische Reich im Gegensatz zu China über lange Zeiten eine gemeinsame Grenze mit dem Partherreich hatte, ist dies nicht überraschend.

Welche Schlüsse lassen sich nun in Bezug auf die Wahrnehmung der Parther als Bindeglied im Handel entlang der Seidenstraße durch Rom und China ziehen? Sowohl römische als auch chinesische Quellen thematisieren erstens, dass nicht sie selbst, sondern andere aus dem Fernhandel Profit zogen. Während die Römer die Parther, ähnlich wie sich selbst, als Konsumenten und nicht als vom Handel profitierende Akteure sahen, zeigen chinesische Quellen die Parther als aktiv auf Gewinn ausgerichtete Händler. Nur eine Herodian-Stelle, an deren Glaubwürdigkeit gezweifelt wird, würde auf eine ähnliche römische Sichtweise hindeuten.

Zweitens unterscheiden sich die Wertungen der Parther in den römischen und chinesischen Quellen deutlich. Während römische Quellen, die sich primär mit den militärischen Auseinandersetzungen zwischen Parthern und Römern beschäftigen, oft negative Topoi aufweisen, sind die chinesischen Quellen um einiges neutraler. Als Beispiel einer negativen Beschreibung kann allenfalls die Darstellung der parthischen Schiffer im „Hou Hanshu“ angeführt werden.

Drittens enthalten römische wie chinesische Quellen geographische Beschreibungen des Partherreichs. Während die chinesischen Beschreibungen für die europäische Geschichtswissenschaft weniger zugänglich sind, da nicht alle verwendeten Bezeichnungen eindeutig geklärt sind, geben vor allem die "Parthischen Stationen“ des Isidor von Charax Aufschluss über wichtige Städte und Wege.

Schließlich muss nochmals betont werden, dass für eine abschließende Bewertung der Parther als Bindeglied im Handel der Seidenstraße auch eine Analyse der Quellen anderer Länder, wie zum Beispiel jener aus Kouchan und Samarkand, durchgeführt werden müsste. Nur durch eine Erweiterung auf diese Quellen kann ein differenziertes Bild der Parther gewonnen werden. Auch eine genauere Analyse des Einflusses der Parther auf ihre Vasallen wäre in diesem Zusammenhang wichtig, da zentrale Handelspunkte, wie Charax Spasinou, in Vasallenreichen lagen. Während die Rolle palmyrenischer Händler in Charax Spasinou und Mesene gut belegt ist, müsste dies für die Parther noch detaillierter untersucht werden.

Zudem wäre es gewinnbringend, die Handelspraktiken der Parther in einen längeren zeitlichen Kontext zu stellen; hier würde sich eine Ausweitung der Untersuchung auf die seleukidische und sassanidische Regierungsepoche anbieten, da sich die wirt- 
schaftlichen Systeme in einer Region nicht schlagartig mit dem Wandel der herrschenden Dynastie ändern, und die Quellenlage für diese Zeiten teilweise besser als für das Partherreich ist.

Generell muss aber festgehalten werden, dass Texte aus Drittländern nur einen subjektiven Blick von außen ermöglichen. Daher wird es wichtig sein, derartige Untersuchungen weiter mit archäologischen Informationen zu untermauern, sobald diese zugänglich sind.

\section{Literatur}

Börm, Henning, Kontinuität im Wandel. Begründungsmuster und Handlungsspielräume der iranischen Monarchie in arsakidischer und sasanidischer Zeit, in: Stefan Rebenich (Hrsg.), Monarchische Herrschaft im Altertum (Schriften des Historischen Kollegs 94), München 2017, S. 545-564.

Caley, Earle R., Chemical Composition of Parthian Coins, in: Numismatic Notes and Monographs 129 (1955), S. 1-104.

Choisnel, Emmanuel, Les Parthes et la Route de la Soie (Collection Centre Asie), Paris 2004

Colledge, Malcolm A. R., The Parthians. Ancient Peoples and Places, Holland 1967.

Dubs, Homer H., The Reliability of Chinese Histories, in: The Far Eastern Quarterly 6 (1946), Heft 1, S. 23-43.

Gabriel, Lisa-Marie, Von der Oase zur antiken Handelsmetropole. Überlegungen zur wirtschaftlichen Rolle und Bedeutung der nahöstlichen Oasenstadt Palmyra im Kontext der römischen Ostexpansion, in: historia.scribere 9 (2017), S. 213-242, [http://historia.scribere.at/, eingesehen 5. 4. 2019.

Golze, Uta/Storm, Kerstin, Chinesische Quellen zum Partherreich, in: Ursula Hackl/Bruno Jacobs/Dieter Weber (Hrsg.), Quellen zur Geschichte des Partherreiches. Textsammlung mit Übersetzungen und Kommentaren, Bd. 3 (Novum Testamentum et orbis antiquus 85), Göttingen u. a. 2010, S. 482-512.

Grajetzki, Wolfram, Greeks and Parthians in Mesopotamia and beyond, 331 BC-224 AD, London 2011.

Hackl, Ursula, Das Partherreich und Rom seit dem 1. Jh. v. Chr., in: Ursula Hackl/Bruno Jacobs/Dieter Weber (Hrsg.), Quellen zur Geschichte des Partherreiches. Textsammlung mit Übersetzungen und Kommentaren, Bd. 1 (Novum Testamentum et orbis antiquus 83), Göttingen u. a. 2010, S. 56-77.

Dies., Handel und Wirtschaft, in: Hackl/Jacobs/Weber (Hrsg.), Quellen, Bd. 1, S. 111-124.

Dies., Schriftquellen zur Geschichte des Partherreichs, in: Hackl/Jacobs/Weber (Hrsg.), Quellen, Bd. 1, S. 21-30. 
Hansen, Valerie, The Silk Road. A New History, Oxford 2012.

Hartmann, Udo, Die Parthischen Stationen des Isidor von Charax: eine Handelsroute, eine Militärkarte oder ein Werk geographischer Gelehrsamkeit?, in: Sabine Müller/Josef Wiesehöfer (Hrsg.), Parthika. Greek and Roman Author's Views of the Arasacid Empire (Classica et Orientalia 15), Wiesbaden 2017, S. 87-125.

Heil, Matthäus, Die Parther bei Tacitus, in: Müller/Wiesehöfer, Parthika, S. 259-278.

Huber, Irene/Hartmann, Udo, 'Denn ihrem Diktat vermochte der König nicht zu widersprechen'. Die Position der Frauen am Hof der Arsakiden, in: Antonio Panaino/Andrea Piras (Hrsg.), Proceedings of the Fifth Conference of Iranian Studies held in Ravenna, 6-11 October 2003 (Ancient \& Middle Iranian Studies 1), Milano 2006, S. 486-517.

Jacobs, Bruno, Die Satrapienverwaltung im Perserreich zur Zeit Darius'III. (Beihefte zum Tübinger Atlas des Vorderen Orients B 87), Wiesbaden 1994.

Ders., Verwaltung,in: Hackl/Jacobs/Weber (Hrsg.), Quellen, Bd. 1, S. 84-100.

Keall, Edward John, Parthian Nippur and Vologases'Southern Strategy. A Hypothesis, in: Journal of the American Oriental Society 95 (1975), Heft 4, S. 620-632.

Lukonin, Vladimir Grigor'evich, Political, Social and Administrative Institutions. Taxes and Trade, in: Iṇsān Yāršātir (Hrsg.), The Cambridge history of Iran, Bd. 3/2, Cambridge 1983, S. $681-746$.

Millward, James A., The Silk Road. A Very Short Introduction (A Very Short Introduction 351), Oxford 2013.

Mittag, Peter Franz, Bactria and Parthia, in: Michel Amandry/Donal Bateson (Hrsg.), A Survey of Numismatic Research 2002-2007, Glasgow 2009, S. 111-116.

Müller, Sabine, Das Bild der Parther bei Trogus-Justin, in: Müller/Wiesehöfer (Hrsg.), Parthika, S. 241-257.

Nickel, Lukas, The First Emperor and sculpture in China, in: Bulletin of School of Oriental and African Studies 76 (2013), Heft 3, S. 413-447.

Olbrycht, Marek Jan, Greeks in the Arsacid Empire, in: Müller/Wiesehöfer (Hrsg.), Parthika, S. 3-27.

Schippmann, Klaus, Grundzüge der parthischen Geschichte (Grundzüge 39), Darmstadt 1980.

Schmitthenner, Walter, Rome and India. Aspects of Universal History during the Principate, in: The Journal of Roman Studies 69 (1979), S. 90-106.

Schuol, Monika, Isidor von Charax und die literarische Gattung der Stathmoi, in: in: Müller/Wiesehöfer (Hrsg.), Parthika, S. 71-85.

Sellwood, David, Parthian Coins, in: Iṇsān Yāršātir (Hrsg.), The Cambridge history of Iran, Bd. 3/1, Cambridge 1983, S. 279-299. 
Stuurman, Siep, Herodotus and Sima Qian. History and the Anthropological Turn in Ancient Greece and Han China, in: Journal of World History 19 (2008), Heft 1, S. 1-40.

Thommen, Lukas, Literarische Texte, in: Hackl/Jacobs/Weber (Hrsg.), Quellen, Bd. 2, S. 22-434.

Weber, Dieter, Parthische Texte, in: Hackl/Jacobs/Weber (Hrsg.), Quellen, Bd. 2, S. 492588.

Wiesehöfer, Josef, Das frühe Persien. Geschichte eines antiken Weltreichs (C. H. Beck Wissen 2107), München 2015.

Ders., Parther und Sasaniden. Imperien zwischen Rom und China, in: Michael Gehler/ Robert Rollinger (Hrsg.), Imperien und Reiche in der Weltgeschichte. Epochenübergreifende und globalhistorische Vergleiche, Bd. 1, Wiesbaden 2014, S. 449-478.

Ders./Müller, Sabine, Einführung, in: Josef Wiesehöfer/Sabine Müller (Hrsg.), Parthika. Greek and Roman Authors'Views of the Arsacid Empire, Wiesbaden 2017, S. VII-XIII.

Wiesehöfer, Josef/Müller, Sabine (Hrsg.), Parthika. Greek and Roman Authors'Views of the Arsacid Empire (Classica et Orientalia 15), Wiesbaden 2017.

Young, Gary K., Rome's Eastern Trade. International Commerce and Imperial Policy 31 BC-AD 305, New York 2011.

\section{Quellen}

Casson, Lionel, The Periplus Maris Erythraei. Text with Introduction, Translation, and Commentary, Princeton 1989.

Fan Xuanling, Jinshu, übersetzt von Uta Golze und Kerstin Storm, herausgegeben von Ursula Hackl/Bruno Jacobs/Dieter Weber, in: Hackl/Jacobs/Weber (Hrsg.), Quellen, Bd. 3, S. 502.

Fan Ye, Hou Hanshu, übersetzt von Uta Golze und Kerstin Storm, herausgegeben von Ursula Hackl/Bruno Jacobs/Dieter Weber, in: Hackl/Jacobs/Weber (Hrsg.), Quellen, Bd. 3, S. 497.

Gaius Plinius Secundus der Ältere, Naturkunde. Buch VI, lateinisch-deutsch, übersetzt und herausgegeben von Kai Brodersen (Sammlung Tusculum), Zürich 1998.

Ders., Naturkunde. Bücher XII/XIII, lateinisch-deutsch, übersetzt und herausgegeben von Roderich König/Gerhard Winkler (Sammlung Tusculum), München 1977.

Herodian, Geschichte des Kaisertums nach Marcus, griechisch-deutsch, übersetzt von Lukas Thommen, in: Hackl/Jacobs/Weber (Hrsg.), Quellen, Bd. 2, S. 174-189.

Sima Qian, Shiji, chinesisch-deutsch, übersetzt von Uta Golze und Kerstin Storm, herausgegeben von Ursula Hackl/Bruno Jacobs/Dieter Weber, in: Hackl/Jacobs/Weber (Hrsg.), Quellen, Bd. 3, S. 487. 
Bettina Darlap ist Studentin der Classica et Orientalia an der Universität Innsbruck. Bettina.Darlap@student.uibk.ac.at

\section{Zitation dieses Beitrages}

Bettina Darlap, Das Partherreich als Bindeglied der Seidenstraße zwischen China und Rom, in: historia.scribere 11 (2019), S. 347-372, [http://historia.scribere.at], eingesehen 17.6.2019 (=aktuelles Datum).

(C) Creative Commons Licences 3.0 Österreich unter Wahrung der Urheberrechte der Autorlnnen. 